\title{
Is preoperative opioid utilization an important factor in predicting phantom limb pain?
}

\author{
Harsha Shanthanna, MD
}

Received: 7 February 2010/ Accepted: 1 March 2010/Published online: 19 March 2010

(C) Canadian Anesthesiologists' Society 2010

\section{To the Editor,}

I was interested in reading the article by Roullet et al. published in the Journal. ${ }^{1}$ In a patient group suffering primarily from peripheral vascular disease, the authors observed that the incidence of phantom limb pain was higher in patients treated preoperatively with opioids. The study raises some questions that merit consideration.

The study assessed the postoperative morphine requirements in patients who were receiving opioids preoperatively in the context of phantom limb pain. However, the patients were included in the study regardless of the reason for analgesic consumption. The knowledge that chronic opioid utilization in such patients is associated with tolerance and hyperalgesia and the need for increased postoperative opioids has been validated by other studies and described in another article in the same issue of the Journal. $^{2}$ However, that knowledge alone cannot be the reason to infer that preoperative opioid consumption increases the risk of phantom limb pain and hence an increased need for postoperative opioids. Increased preamputation pain is also known to be a significant factor in determining the likelihood of phantom pain. This aspect was also observed in the same study and may have been the primary reason for the increased incidence of phantom pain in this cohort.

Ketamine has been used effectively for phantom limb pain. $^{3}$ Although ketamine was used in this study, its effect on the incidence of phantom limb pain was not evaluated in either group.

One final issue to consider is whether sciatic nerve blockade alone was adequate to achieve complete sensory

H. Shanthanna, MD ( $₫)$

McMaster University, Hamilton, ON, Canada

e-mail: harshamd@gmail.com blockade during amputation. Wilson et al. achieved better results than observed in other studies employing regional analgesia and claim this difference was due to the "dense block of sensory input" by combining both spinal and epidural analgesia. ${ }^{4}$ An adequate reduction of the massively increased sensory input that occurs during amputation requires a block dense enough to prevent spinal or supraspinal responses. It has also been shown that local anesthetic administered via the epidural route does not abolish the peripheral sensory input completely. ${ }^{5}$ Sciatic nerve block may not be able to block all of the painful and non-painful impulses elicited during amputation. Functional magnetic resonance imaging has shown that electrical activity well below the threshold required to activate spinal responses may evoke neuronal impulses in the brainstem. ${ }^{6}$

Competing interests None declared.

\section{References}

1. Roullet S, Nouette-Gaulain K, Biais M, et al. Preoperative opioid consumption increases morphine requirement after leg amputation. Can J Anesth 2009; 56: 908-13.

2. Richebe $P$, Beaulieu P. Perioperative pain management in patient treated with opioids: continuing professional development. Can J Anesth 2009; 56: 969-81.

3. Nikolajsen L, Hansen CL, Nielsen J, Keller J, Arendt-Nielsen L, Jensen TS. The effect of ketamine on phantom pain: a central neuropathic disorder maintained by peripheral input. Pain 1996; 67: 69-77.

4. Wilson JA, Nimmo AF, Fleetwood-Walker SM, Colvin LA. A randomised double blind trial of the effect of pre-emptive epidural ketamine on persistent pain after lower limb amputation. Pain 2008; 135: 108-18.

5. Loughman BA, Fennelly ME, Henley M, Hall GM. The effects of differing concentrations of bupivacaine on the epidural 
somatosensory evoked potential after posterior tibial nerve stimulation. Anesth Analg 1995; 81: 147-51.

6. Lilja J, Endo T, Hofstetter $C$, et al. Blood oxygenation leveldependent visualization of synaptic relay stations of sensory pathways along the neuroaxis in response to graded sensory stimulation of a limb. J Neurosci 2006; 26: 6330-6.

\section{Reply}

Thank you for the opportunity to address the questions raised by Dr. Harsha Shanthanna regarding our recently published study. ${ }^{1}$

The fact that our patients were included in the study regardless of the reason for analgesic consumption could add a bias in the determination of the greatest risk factor for phantom limb pain-chronic preoperative pain in the limb or chronic morphine consumption. In the event of amputation due to peripheral vascular disease, the most frequent pain, if present, is limb pain, which is subischemic. It is difficult to dissociate pain and analgesic consumption. The latter could be insufficient, inappropriate, or ineffective for treating preoperative pain. Therefore, the question is whether preoperative opioid consumption truly reflects preoperative pain, and which has the greater effect on the incidence of phantom limb pain.

Since ketamine was used in both groups, we could not assess its possible effect on the incidence of phantom limb pain. Ketamine has proved effective for the treatment ${ }^{2,3}$ but not for the prevention of phantom limb pain. ${ }^{4}$

The physiopathology of phantom limb pain is complex and includes peripheral, spinal, and supraspinal mechanisms. ${ }^{5}$ Sciatic nerve block alone is probably insufficient to block all stimuli elicited during leg amputation; therefore, we think that it should be performed with multimodal analgesia. Continuous sciatic nerve block with the insertion of a catheter could be started several days before surgery in order to diminish preoperative pain. The block could be continued during amputation as part as the anesthesia protocol, and finally, it could be used for postoperative analgesia.

Competing interests None declared.

\section{References}

1. Roullet $S$, Nouette-Gaulain K, Biais $M$, et al. Preoperative opioid consumption increases morphine requirement after leg amputation. Can J Anesth 2009; 56: 908-13.

2. Eichenberger $U$, Neff $F$, Sveticic $G$, et al. Chronic phantom limb pain: the effects of calcitonin, ketamine, and their combination on pain and sensory thresholds. Anesth Analg 2008; 106: 1265-73.

3. Nikolajsen L, Hansen CL, Nielsen J, Keller J, Arendt-Nielsen L, Jensen TS. The effect of ketamine on phantom pain: a central neuropathic disorder maintained by peripheral input. Pain 1996; 67: 69-77.

4. Wilson JA, Nimmo AF, Fleetwood-Walker SM, Colvin LA. A randomised double blind trial of the effect of pre-emptive epidural ketamine on persistent pain after lower limb amputation. Pain 2008; 135: 108-18.

5. Flor $H$. Phantom-limb pain: characteristics, causes, and treatment. Lancet Neurol 2002; 1: 182-9.

Stéphanie Roullet, MD

François Sztark, MD, PhD

CHU Pellegrin, Bordeaux, France 